(c) Elsevier/INRA

\title{
Méthodes d'exploitation de données concernant les débits sanguins mesurés au niveau des viscères et du train-arrière chez la brebis
}

\author{
A Isserty, I Ortigues \\ INRA-Theix, laboratoire croissance et métabolismes des herbivores, \\ 63122 Saint-Genès-Champanelle, France
}

(Reçu 18 décembre 1993; accepté le 17 juin 1994)

\begin{abstract}
Résumé - Deux méthodes d'exploitation de valeurs de débits sanguins sont présentées. Elles cherchaient à réduire la part de variabilité i) liée à des aspects méthodologiques lorsque les débits splanchniques étaient mesurés par dilution ( $\mathrm{PAH})$, et ii) liée à l'activité physique pour les débits aortiques mesurés par sonde ultrasonique. Six brebis multicathétérisées, alimentées d'abord à l'entretien puis à $50 \%$ de l'entretien, ont été utilisées. Les débits splanchniques observés étaient très variables lorsque le $\mathrm{PAH}$ était infusé en veine mésentérique seule (moyenne des CV : 17\%). La variabilité a été diminuée à la fois par une infusion de $\mathrm{PAH}$ en veine mésentérique et en veine ruminale ( $\mathrm{CV}=11 \%)$, par des corrections rigoureuses sur les valeurs instantanées de débit $(C V=13,5 \%)$ et par une combinaison des 2 (CV $=9,5 \%)$. Les corrections n'ont pas changé les conclusions de l'analyse de variance sur l'effet traitement mais elles ont permis de réduire la variance résiduelle et de supprimer les débits négatifs de l'artère hépatique. Au niveau du train-arrière, les corrections ont permis de ramener les débits aortiques des animaux à un même état «debout calme». Elles ont diminué la variabilité journalière ( 22 à $8 \%$ ) et permis de mettre en évidence un effet traitement qui n'apparaissait pas sur les données observées.
\end{abstract}

débits sanguins / viscères / train-arrière / PAH / ultrasonique

Summary - Methods of analysis of splanchnic and aortic blood flow data obtained in ewes. Two methods of analysis of blood flow data are presented. They are aimed at reducing: 1) the methodological variability associated with splanchnic blood flows measured by dilution (PAH) and 2) the variability of aortic blood flows measured ultrasonically associated with physical activity. Six multicatheterized ewes were used; they were first fed at maintenance and then at half-maintenance. Observed splanchnic blood flows were very variable when $P A H$ was infused in mesenteric vein only (average $C V=17 \%$ ). Variability was first reduced by a $P A H$ infusion via both a mesenteric and a ruminal vein ( $C V=$ $11 \%)$, second by rigorous corrections on instantaneous blood flow data ( $C V=13.5 \%)$, or by a combination of both ( $\mathrm{CV}=9.5 \%)$. Corrections did not modify the conclusions of the analysis of variance concerning the treatment effect, but reduced the residual variance and eliminated the negative hepatic artery blood flow values. At the hindquarters level, corrections allowed us to approach the aortic blood flows of animals in a similar 'quietly standing' state. They decreased the daily variability (from 22 to $8 \%)$ and enabled the detection of a treatment effect, which was not shown by observed data. 


\section{INTRODUCTION}

Dans le domaine du métabolisme énergétique, un nombre croissant d'études vise à compléter les connaissances acquises au niveau de l'animal entier par des mesures réalisées directement au niveau de tissus corporels spécifiques (Ortigues, 1991). Les dépenses énergétiques de ces tissus peuvent être mesurées in vitro (coupes de tissu, cellules isolées, tissu isolé perfusé) ou in vivo chez des animaux équipés de cathéters sanguins (Katz et Bergman, 1969). L'approche in vivo présente l'avantage majeur de permettre la quantification des phénomènes étudiés en conservant le contexte «animal». Par contre ce type d'étude nécessite de déterminer les bilans de gaz sanguins $\left(\mathrm{O}_{2}\right.$, $\mathrm{CO}_{2}$ ) à travers les différents tissus impliquant la mesure de débits sanguins. Cette dernière peut s'effectuer soit par dilution, soit par débitmétrie électromagnétique ou ultrasonique (à l'aide de sondes posées autour des vaisseaux considérés).

Au niveau splanchnique, les débits sanguins présentent une forte variabilité liée à une régulation physiologique. Par ailleurs, lorsque les débits sont mesurés par dilution, cette variabilité est accrue du fait de problèmes méthodologiques (mauvais mélange du marqueur dans le sang, choix du site d'infusion, mauvais placement de cathéters, problème de dosage). De même au niveau du train-arrière, une forte variabilité des débits sanguins existe. Elle est essentiellement due à l'activité physique spontanée de l'animal (position debout ou couchée, agitation). Des différences d'activité entre animaux ou entre jours de prélèvement peuvent alors biaiser les comparaisons entre traitements (Harris et al, 1989). Par ailleurs, les études in vivo sont très lourdes financièrement, en temps et en main d'œuvre. Le nombre d'animaux étudiés ne peut donc pas être facilement démultiplié, ce qui exige d'exploiter au mieux les résultats obtenus.
Dans une expérimentation sur l'adaptation du métabolisme énergétique de certains tissus (tube digestif, foie, train-arrière) à la sous-alimentation chez des brebis adultes, non lactantes, non gestantes, les débits splanchniques ont été mesurés par une méthode de dilution utilisant l'acide para-aminohippurique (PAH), alors que les débits au niveau du train-arrière étaient mesurés par débitmétrie ultrasonique. Cette étude a fait l'objet de travaux méthodologiques et métaboliques.

Cet article rapporte les aspects méthodologiques. L'objectif premier de ce travail est de présenter et d'analyser un mode d'exploitation des résultats de débits sanguins qui cherche à réduire, i) dans la mesure des débits splanchniques, la part de variabilité qui est liée à des aspects méthodologiques (en effet, dans le cadre de l'expérience sur la sous-alimentation mentionnée précédemment, nous avons eu la possibilité de tester la méthode proposée par Ortigues et al (1994) concernant le dépouillement des résultats de débits splanchniques et le choix du site d'infusion de PAH dans d'autres conditions nutritionnelles), et, ii) dans la mesure des débits au niveau train-arrière, la part de variabilité liée à l'activité physique. Le second objectif est de tester les conséquences du traitement des données sur la détection statistique d'un effet traitement (effet sous-alimentation). Les effets de la sous-alimentation sur les dépenses énergétiques des tissus sont rapportés par Ortigues et Durand (1994).

\section{MATÉRIEL ET MÉTHODES}

\section{Animaux}

Six brebis adultes (désignées par des lettres alphabétiques $A, B, C, L, M, O$ ), non gestantes, non lactantes, de race Romanov $x$ Limousine, de poids vif (PV) moyen initial de $50,2 \mathrm{~kg} \pm 3,06$ (écart type) ont été utilisées. Elles avaient été 
initialement sélectionnées pour leur docilité et leur caractère calme. Après 3 sem d'adaptation à l'environnement (logement, manipulations) et au régime, elles étaient équipées chirurgicalement de cathéters sanguins placés au niveau de la veine porte (VP), d'une veine sus-hépatique (VSH), de la veine cave (VC), des veines mésentérique (VM) et ruminale (VR) et d'une artère mésentérique (AM) selon la méthode décrite par Ortigues et al (1994). Une sonde débitmétrique ultrasonique a été placée autour de l'aorte postérieure (Ao) à l'aplomb de l'extrémité du cathéter cave, afin que les mesures de débits et de métabolites se rapportent à la même zone de tissus irrigués.

Les brebis étaient logées dans une pièce climatisée, à éclairage permanent, dans des cages individuelles sur caillebotis.

\section{Alimentation}

Le régime était composé uniquement de foin de montagne à base de dactyle de qualité moyenne et d'une valeur énergétique estimée en début d'expérience à 2,10 Mcal d'énergie métabolisable $(E M) / k g$ matière sèche. Une supplémentation minérale et vitaminique (à raison de $10 \mathrm{~g} / \mathrm{j}$ de compléments en minéraux et vitamines $A, D$ et $E$ et d'un oligo-bloc à volonté) a assuré une couverture des besoins théoriques des brebis pendant toute l'expérience. Les brebis étaient alimentées toutes les $3 \mathrm{~h}$ (soit 8 repas par jour) à l'aide de distributeurs automatiques d'aliment. Les animaux ont reçu successivement 2 niveaux alimentaires. Tout d'abord le niveau d'entretien (335 kJ EM/kg PV initial $^{0,75 / j)}$ a été distribué pendant 3 sem (sem -3 à -1 ) après la période de récupération post-chirurgicale. Puis la ration a été diminuée de $50 \%$ (période de sous-alimentation), et est restée inchangée pendant 5 sem $($ sem +1 à +5$)$.

\section{Mesures}

Des mesures de débits sanguins ont été effectuées une fois par semaine au cours des 2 dernières semaines d'entretien (sem -2 et -1 ) puis chaque semaine au cours de la période de sousalimentation (sem +1 à +5$)$.

Les débits en VP et VSH ont été mesurés par l'infusion continue (à l'aide d'une pompe à seringues) d'un marqueur (acide para-aminohip- purique, $\mathrm{PAH}$ ), au taux de $0,2 \mathrm{mg} \mathrm{PAH} / \mathrm{mn} / \mathrm{kg}$ PV (Ortigues et al, 1994). L'infusion était effectuée simultanément en VM et VR, la quantité totale infusée étant répartie pour moitié dans chaque vaisseau. Avant le début de l'infusion, une surcharge de $\mathrm{PAH}(3,75 \mathrm{mg} / \mathrm{kg} \mathrm{PV})$ était injectée en VM afin d'atteindre rapidement un plateau d'équilibre des concentrations en PAH dans le sang. $L$ 'infusion qui commençait $\mathbf{4 5}$ min avant un repas était poursuivie pendant les $6 \mathrm{~h}$ suivant le repas, soit 2 cycles alimentaires. Les prélèvements sanguins en VP, VSH et AM débutaient $15 \mathrm{~min}$ après le repas puis étaient effectués toutes les $30 \mathrm{~min}$ pendant 2 cycles alimentaires, soit un total de 12 prélèvements par vaisseau. Les échantillons de sang prélevés sur héparine étaient conservés à $-20^{\circ} \mathrm{C}$. Le dosage du $\mathrm{PAH}$ a été effectué sur sang total d'après la méthode d'Huntington (1982).

Au niveau du train-arrière, les débits ont été mesurés à l'aide d'une sonde ultrasonique de type 12R (Transonic Systems Inc, Ithaca, NY, USA). Le débitmètre était relié à un micro-ordinateur dont le programme de saisie permettait d'intégrer 1770 mesures instantanées par minute, ceci pendant les $6 \mathrm{~h}$ de mesure. Pendant la durée des mesures, les animaux étaient attachés tout en ayant la possibilité de se coucher sauf pendant les prélèvements sanguins et les $4 \mathrm{~min}$ qui les précédaient où ils étaient debout. Le comportement de l'animal (couché, agitation) ainsi que les interventions sur celui-ci étaient notés.

\section{Méthodes de calculs et exploitation des données}

Les débits splanchniques observés (VP et VSH) ont été calculés en fonction du taux d'infusion et des différences de concentration en $\mathrm{PAH}$ entre les vaisseaux afférents et efférents aux tissus :

débits sanguins VP ( $/ / \mathrm{min})$

Vitesse infusion $(1 / \mathrm{min}) \times$ conc PAH infusé $(\mathrm{mg} / \mathrm{l})$

(conc PAH VP - conc PAH AM) (mg/l)

débits sanguins VSH ( $/ / \mathrm{min})$

Vitesse infusion ( $/ / \mathrm{min}) \times$ conc PAH infusé $(\mathrm{mg} / \mathrm{l})$ $=$

(conc PAH VSH - conc PAH AM) (mg/l)

avec conc $\mathrm{PAH}$ infusé = concentration de $\mathrm{PAH}$ dans la solution infusée ; conc PAH VP = concen- 
tration de $\mathrm{PAH}$ en veine porte; conc $\mathrm{PAH} \mathrm{VSH}=$ concentration de $\mathrm{PAH}$ en veine sus-hépatique ; conc $\mathrm{PAH} A M=$ concentration de $\mathrm{PAH}$ en artère mésentérique.

Les valeurs instantanées des débits splanchniques ont été soit utilisées sans aucune correction préalable (débits observés), soit traitées selon la méthode décrite par Ortigues et al (1994) (débits corrigés). Ces auteurs ont déterminé que, dans des conditions optimales de mesure (infusion de PAH en VM et VR), les coefficients de variation (CV) des débits splanchniques étaient en moyenne de $11 \%$. Des CV supérieurs à $11 \%$ indiquaient des difficultés d'ordre méthodologique. Ainsi, lorsque les CV journaliers (CVj) étaient supérieurs à $11 \%$, il était préconisé d'éliminer au maximum 2 points (sur les 12 valeurs obtenues par journée de prélèvements, par animal et par vaisseau) afin de réduire de $\mathrm{CVj}$ à une valeur proche de celle de référence estimée à $11 \%$. Dans le cas où le nombre de prélèvements était inférieur à 9 , soit $75 \%$ du nombre total de prélèvements (Casse, 1990), à cause d'un mauvais fonctionnement de cathéters, aucune correction n'était apportée. Les valeurs instantanées observées ou corrigées ont ensuite été utilisées pour calculer les débits sanguins moyens journaliers, accompagnés de leurs $\mathrm{CVj}$.

Les débits de l'artère hépatique $(\mathrm{AH})$ ont été obtenus par différence entre le débit moyen journalier de la VSH et le débit moyen journalier de la VP. Le calcul a été réalisé à la fois sur les débits moyens journaliers observés et corrigés. Le calcul de la contribution de l'AH au débit hépatique total a aussi été effectué d'après les débits moyens journaliers observés et corrigés.

Les débits aorte observés, enregistrés toutes les minutes pendant $6 \mathrm{~h}$, ont été traités afin de prendre en compte la part de variabilité liée à l'activité physique (position debout-couchée, agitation momentanée). Celle-ci peut être importante, et peut surtout être très différente entre les animaux ou entre les jours de prélèvements en termes de fréquence et de durée. Les corrections appliquées avaient pour but de ramener les débits Ao de tous les animaux à ceux correspondant à un même état "debout calme" afin que toutes les comparaisons entre animaux et entre traitements soient plus fiables. Ces corrections ont été faites en 3 temps. Tout d'abord, les $6 \mathrm{~h}$ d'enregistrement ont été divisées en tranches de $30 \mathrm{~min}$ à partir du premier repas. Les valeurs liées à une agitation importante observée (ex: animal qui se gratte) et d'une durée supérieure à 3 min ont été éliminées. Puis, sur les valeurs restantes et après avoir exclu momentanément les débits mesurés lorsque l'animal était couché, ont été calculés la moyenne et l'intervalle de confiance unilatéral $(P<0,05)$ des débits "debout" par tranches de 30 min. Les valeurs supérieures à la limite supérieure de l'intervalle de confiance ont été éliminées. L'hypothèse était que ces valeurs correspondaient à des périodes d'agitation intermédiaire. Enfin, les débits correspondant à une position couchée ont été majorés par la différence (débit "debout" - débit "couché") calculée autour de chaque période "couché». Le débit moyen journalier en Ao et le CVj qui lui est associé ont été calculés en moyennant d'une part les valeurs de débits et d'autre part des coefficients de variation obtenus par tranches de $30 \mathrm{~min}$.

\section{Analyses statistiques}

Une première analyse statistique a été réalisée afin de tester l'influence du traitement des données sur les débits moyens journaliers splanchniques et aortiques ainsi que sur les variabilités journalières ou hebdomadaires. Dans ce but, les débits moyens journaliers observés et corrigés ont été comparés par test $t$ apparié. Par ailleurs, la variabilité journalière a été testée en comparant les CVj correspondant aux données observées et corrigés, sur la base de leurs intervalles de confiance calculés selon Sokal et Rohlf (1969). Enfin, l'influence du traitement des données sur la variabilité inter-animal au sein d'une même semaine de mesures a été testée en comparant (test $F$ ) les écarts types (ET) associés aux débits moyens hebdomadaires observés ou corrigés.

Une deuxième analyse statistique a permis de tester l'influence du site d'infusion du PAH (VM seule ou VM + VR) sur la variabilité des débits splanchniques instantanés mesurés au sein d'une même journée. Pour cela, la comparaison des $C V j$ a été effectuée comme précédemment.

Une troisième analyse statistique concernait l'effet de la sous-alimentation et de la durée de celle-ci sur les débits splanchniques et aortiques. Un test d'analyse de variance à 2 facteurs fixes (animaux, semaines) a été employé pour traiter d'une part les données observées et d'autre part les données corrigées. Les variances résiduelles ont été comparées par test $F$ pour vérifier si les corrections apportées aux débits observés avaient modifié cette analyse. Les résultats physiologiques 
concernant les effets de la sous-alimentation sont rapportés par Ortigues et Durand (1994).

\section{RÉSULTATS}

\section{Cathéters}

Le cathéter en VR n'a fonctionné sur toute la période de mesure que chez 3 animaux $(A, B, L)$, ce qui a permis de mettre en évidence l'importance du site d'infusion du $\mathrm{PAH}$ sur la variabilité des résultats. Aucun résultat n'était disponible en VSH pour la brebis $A$ suite à un mauvais placement du cathéter, d'où l'absence de débits en $\mathrm{AH}$ pour ce même animal. De plus, chez les animaux $A, B, L$, les 12 prélèvements en VP n'ont pas pu être effectués en semaine 5 suite à un fonctionnement irrégulier des cathéters.

\section{Débits splanchniques}

\section{Débits observés}

Une alimentation en continu a permis d'avoir des animaux en état nutritionnel stable, sans variations significatives des débits par rapport à l'heure du repas (résultats non publiés). Les 12 valeurs instantanées de débits splanhniques obtenues toutes les 30 min sur une même journée de mesure peuvent donc être considérées comme des répétitions permettant d'estimer la moyenne journalière.

Pour les 2 sem d'entretien, les débits sanguins en VP (tableau I) et en VSH (tableau II) étaient en moyenne respectivement de $2,35 \mathrm{l} / \mathrm{min}$ et de $2,63 \mathrm{l} / \mathrm{min}$. Ils ont chuté respectivement à $1,85 \mathrm{l} / \mathrm{min}$ et $2,24 \mathrm{l} / \mathrm{min}$ en première semaine de sousalimentation, soit une baisse de $21 \%$ et $15 \%$. Pendant les 4 dernières sem de sousalimentation, les débits en VP et VSH sont restés stables à des valeurs moyennes de $1,91 \mathrm{l} / \mathrm{min}$ en VP et $2,13 \mathrm{l} / \mathrm{min}$ en VSH.

Pour les semaines d'entretien, le débit moyen calculé de l'AH était de 0,35 l/min, sa contribution au débit hépatique était de $13 \%$ en moyenne (tableau III). Pendant la $1^{\mathrm{re}}$ sem de sous-alimentation le débit moyen et la contribution moyenne de l'AH sont passés respectivement à $0,41 \mathrm{l} / \mathrm{min}$ et $17 \%$. Trois valeurs de débit moyen journalier et 3 valeurs de contribution moyenne de l'AH étaient négatives. Les faibles contributions observées de I'AH au débit hépatique total en semaines 2 et 5 étaient attribuables à un animal $(B)$ qui présentait des débits $A H$ négatifs.

Les CVj associés aux débits journaliers obervés étaient en moyenne de $14 \%$ ( $7 \%$ à $31 \%)$ en VP et $14 \%(6 \%$ à $32 \%)$ en VSH (tableaux I et II). En VP, une majorité de CVj (57\%) étaient supérieurs à $11 \%$, de même qu'en VSH (58\%).

\section{Influence du traitement des données sur les valeurs des débits splanchniques}

Sur l'ensemble des animaux, les corrections appliquées ont baissé non significativement les débits en VP et VSH de l'ordre de 2,4 et $3,5 \%$ en moyenne respectivement (tableaux I et II). Par ailleurs, au sein de chaque semaine, la variabilité inter-animal n'a pas été réduite de manière significative.

Au niveau de I'AH (tableau III), les corrections apportées aux débits en VP et VSH $n$ 'ont pas réduit de manière significative la variabilité inter-animal au sein d'une même semaine en ce qui concerne les débits $\mathrm{AH}$ et la contribution de I'AH au débit hépatique total. Elles ont néanmoins permis de supprimer les valeurs négatives.

\section{Corrections apportées en fonction du site d'infusion}

Les débits splanchniques observés étaient associés à une variabilité journalière beau- 


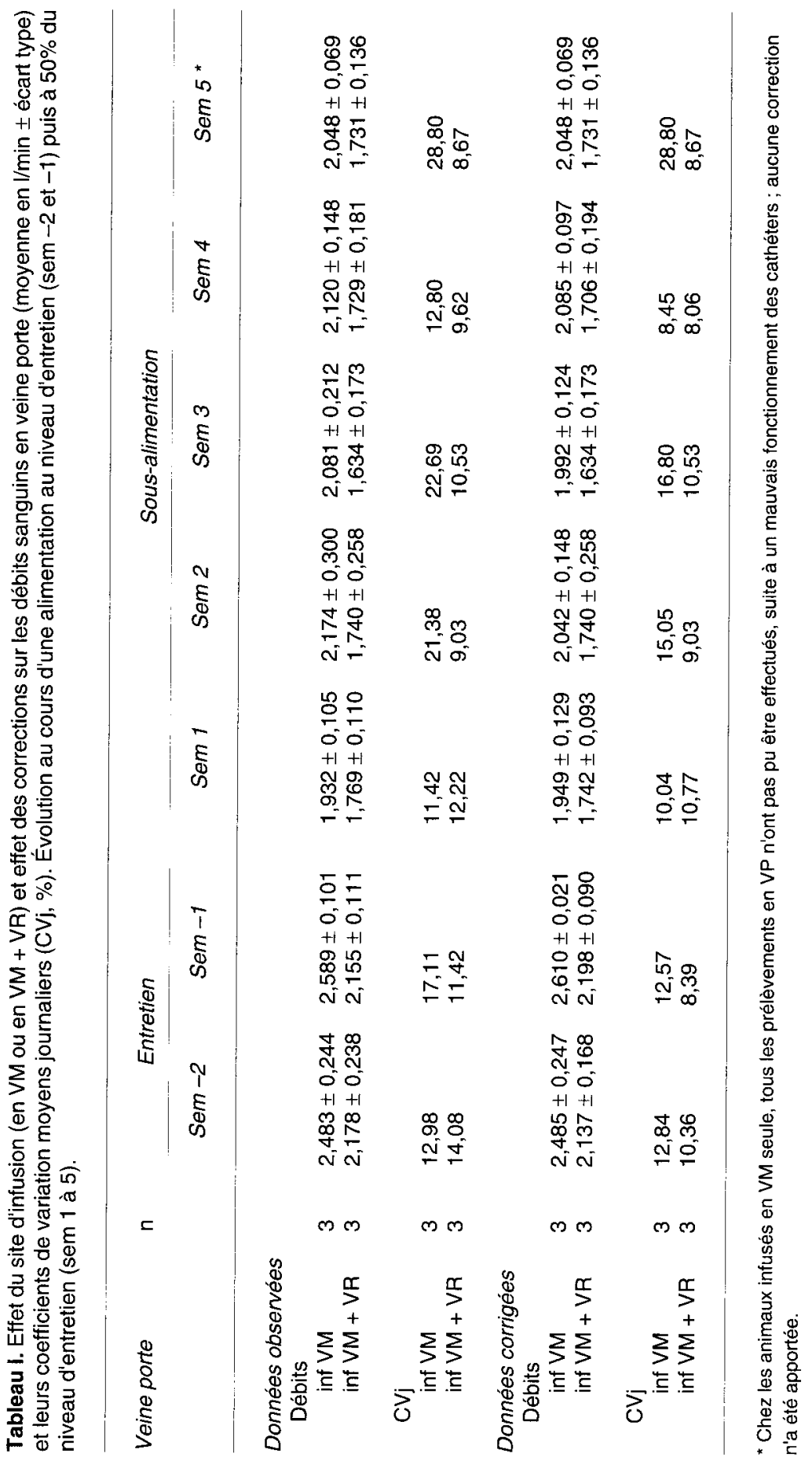




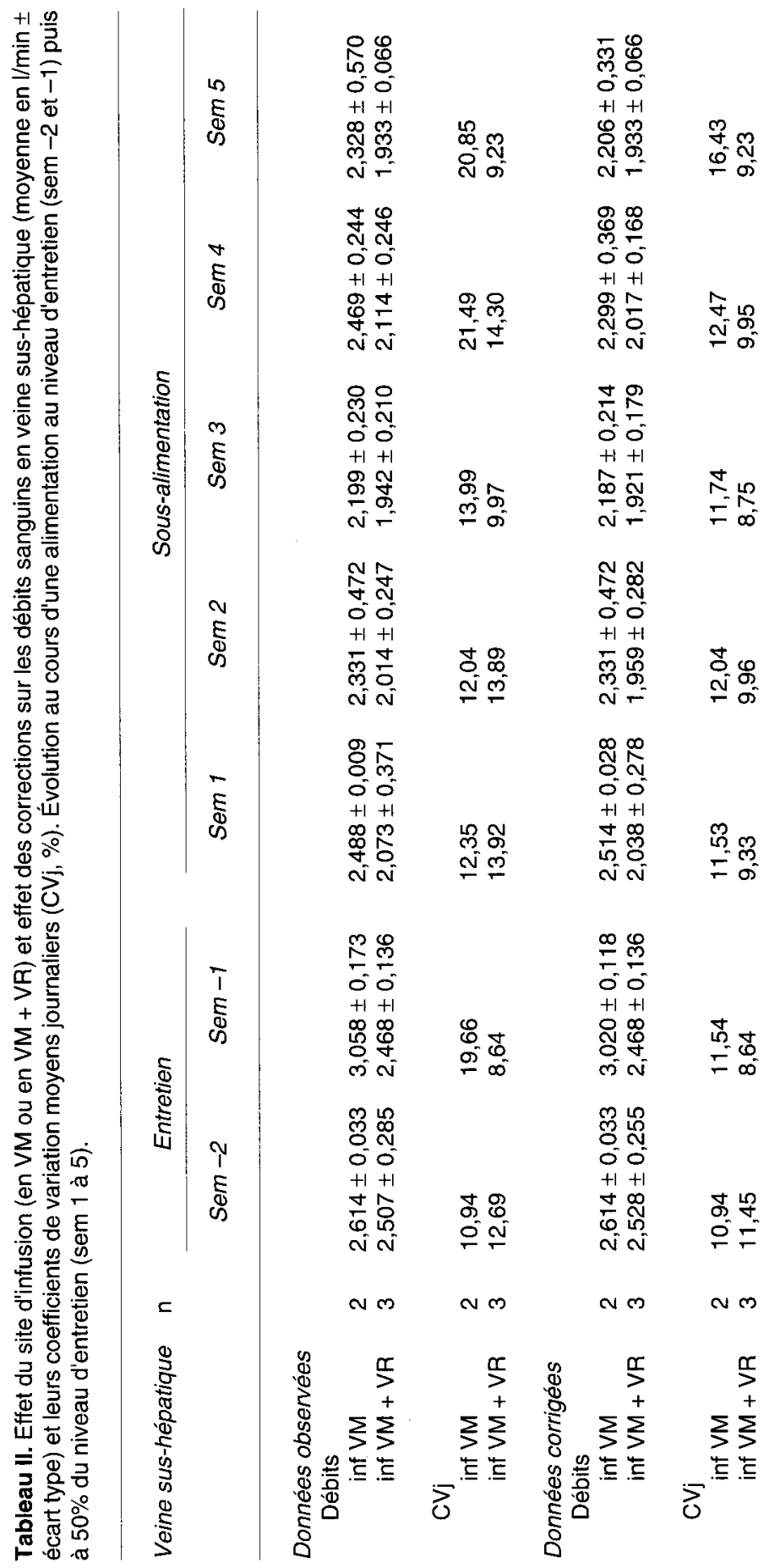




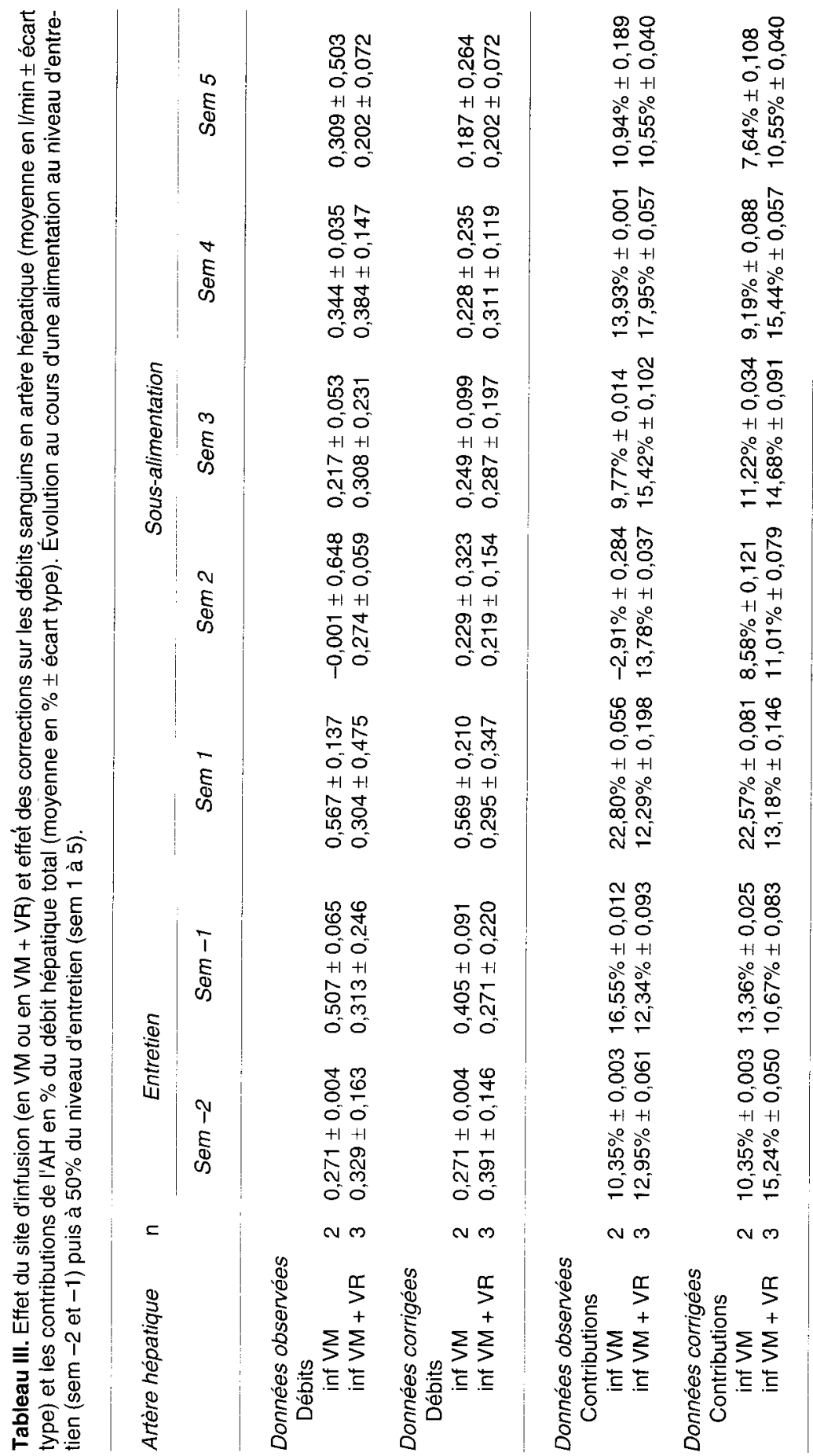


coup plus importante lorsque l'infusion de $\mathrm{PAH}$ était effectuée en un seul site (VM seule) plutôt qu'en 2 sites (VM + VR) (tableau IV). En effet, les CVj observés obtenus chez les animaux infusés en VM seule étaient en moyenne de $18 \%(9 \%$ à $31 \%)$ en VP et de $17 \%(7 \%$ à $32 \%)$ en VSH. Chez les brebis infusées en VM + VR, ils n'étaient plus que de $11 \%(7 \%$ à $21 \%$ ) en VP et de $12 \%$ ( $6 \%$ à $22 \%$ ) en VSH, ce qui représente une baisse respective de $40 \%$ et $29 \%$.

La double infusion a ainsi permis de diminuer très nettement le nombre de données supprimées (tableau IV). Ainsi en ce qui concerne la VP, chez les animaux infusés en VM seule, $59 \%$ (soit plus de la moitié) des débits moyens journaliers ont été corrigés, avec élimination de $9 \%$ des valeurs instantanées de débit. Ces corrections ont permis de baisser les CVj de 18 à $14 \%$ en moyenne. En revanche, chez les animaux infusés en VM + VR, seulement $24 \%$ des débits moyens VP journaliers ont été corri- gés avec une élimination de $2,4 \%$ des valeurs instantanées. Du fait de ces faibles corrections, la moyenne des CVj n'a que peu baissé (de $11 \%$ à $9 \%$ ).

En ce qui concerne la VSH, chez les animaux infusés en VM seule $54 \%$ (soit plus de la moitié) des débits moyens journaliers ont été corrigés, avec une élimination de $7,3 \%$ des valeurs instantanées. Ces corrections ont permis de baisser les $\mathrm{CVj}$ de $17 \%$ à $13 \%$ en moyenne. En revanche, chez les animaux infusés en VM + VR, seulement $33 \%$ des débits moyens VSH journaliers ont été corrigés avec une élimination de $4 \%$ des valeurs instantanées, les CVj passant de 12 à $10 \%$.

\section{Conséquences des corrections sur l'effet traitement}

L'effet traitement avant et après correction est rapporté dans le tableau $\mathrm{V}$. La détection de l'effet traitement est restée inchangée au niveau de la VP, alors qu'elle est légèrement

Tableau IV. Récapitulatif des corrections apportées d'une part aux débits splanchniques mesurés à partir d'animaux infusés en VM $(n=3)$ ou à partir d'animaux infusés en VM et VR $(n=3)$, et d'autre part aux débits aortiques ${ }^{a}$.

$\begin{array}{cccc}\begin{array}{c}\text { Nombre de valeurs } \\ \text { instantanées de }\end{array} & \begin{array}{c}\text { Nombre de débits } \\ \text { moyens journaliers }\end{array} & \text { Moyennes des CV journaliers } \\ \text { débit sanguin } & \text { corrigés (\%) } & \text { Avant } & \text { Après } \\ \text { supprimées }(\%) & & \text { correction } & \text { correction }\end{array}$

\begin{tabular}{|c|c|c|c|c|c|}
\hline \multicolumn{6}{|l|}{ Débits splanchniques } \\
\hline 3 animaux infusés & VP & 9,0 & 59 & 17,93 & 14,43 \\
\hline en VM seule & VSH & 7,3 & 54 & 16,64 & 12,53 \\
\hline 3 animaux infusés & VP & 2,4 & 24 & 10,80 & 9,40 \\
\hline en VM + VR & VSH & 4,0 & 33 & 11,80 & 9,61 \\
\hline
\end{tabular}

Débits aortiques

$\begin{array}{llllll}6 \text { animaux } & \text { Ao } & 25,8 & 100 & 22,03 & 8,03\end{array}$

a Les méthodes de corrections des débits splanchniques d'une part et des débits aortiques d'autre part sont présentées dans les Matériels et méthodes. 
améliorée au niveau de la VSH. La variance résiduelle a été réduite au niveau VP $(-51 \%$, $P<0,05)$, et au niveau VSH $(-23 \%, N S)$.

Au niveau de I'AH, aucun effet significatif du traitement n'a été détecté sur les débits après analyse de variance effectuée d'une part sur les données observées et d'autre part sur les données corrigées (tableau V). Un test $F$ a néanmoins montré que la variance résiduelle a été diminuée de manière significative $(P<0,05)$.

\section{Débits aortiques}

\section{Débits observés}

Au niveau d'entretien, les débits sanguins en Ao étaient en moyenne de $0,55 \mathrm{l} / \mathrm{min}$ (tableau VI). Pendant la $1^{\text {re }}$ semaine de sous-alimentation ils ont chuté à $0,48 \mathrm{l} / \mathrm{min}$ soit une baisse de $12 \%$ et se sont stabilisés à $0,50 \mathrm{l} / \mathrm{min}$ pendant les 4 dernières sem de sous-alimentation. Les CVj observés étaient en moyenne de $22 \%$ ( 10 à $44 \%$ ).

Sur un des animaux (A) les débits Ao n'ont pas pu être enregistrés par voie informatique, seul un enregistrement graphique a pu être effectué. Les débits ont alors été déterminés à partir d'une lecture ponctuelle toutes les minutes. Les CVj observés pour cet animal étaient nettement plus élevés que pour les autres animaux.

\section{Influence des corrections sur les valeurs de débits sanguins et leur variabilité}

Les débits sanguins au niveau de l'Ao peuvent être multipliés par 2 ou 3 lors d'une activité physique particulière. L'élimination des valeurs instantanées liées à l'activité physique a touché en moyenne $26 \%$ de l'en-

Tableau V. Résultats (effets animal et traitement) de l'analyse de variance à 2 facteurs réalisée sur les débits VP, VSH, AH et Ao bruts et corrigés a.

\begin{tabular}{|c|c|c|c|c|c|}
\hline & \multicolumn{2}{|c|}{ Données brutes } & \multicolumn{2}{|c|}{ Données corrigées } & \multirow[t]{2}{*}{$d d l$} \\
\hline & Variance & Probabilité & Variance & Probabilité & \\
\hline \multicolumn{6}{|l|}{$V P$} \\
\hline Effet animal & 0,3182 & 0,0001 & 0,2764 & 0,0001 & 5 \\
\hline Effet traitement & 0,2842 & 0,0001 & 0,3409 & 0,0001 & 6 \\
\hline Variance résiduelle & 0,0249 & & 0,0122 & & 30 \\
\hline \multicolumn{6}{|l|}{ VSH } \\
\hline Effet animal & 0,3910 & 0,001 & 0.3414 & 0,0001 & 4 \\
\hline Effet semaine & 0,2897 & 0,001 & 0,3304 & 0,0001 & 6 \\
\hline Variance résiduelle & 0,0480 & & 0,0369 & & 24 \\
\hline \multicolumn{6}{|l|}{$A H$} \\
\hline Effet animal & 0,1403 & 0,06 & 0,0953 & 0,02 & 4 \\
\hline Effet semaine & 0,0345 & 0,67 & 0,0201 & 0,53 & 6 \\
\hline Variance résiduelle & 0,0509 & & 0,0230 & & 24 \\
\hline \multicolumn{6}{|l|}{$v C$} \\
\hline Effet animal & 0,0236 & 0,0001 & 0,0236 & 0,0001 & 5 \\
\hline Effet semaine & 0,0040 & 0,11 & 0,0056 & 0,01 & 6 \\
\hline Variance résiduelle & 0,0020 & & 0,0013 & & 30 \\
\hline
\end{tabular}




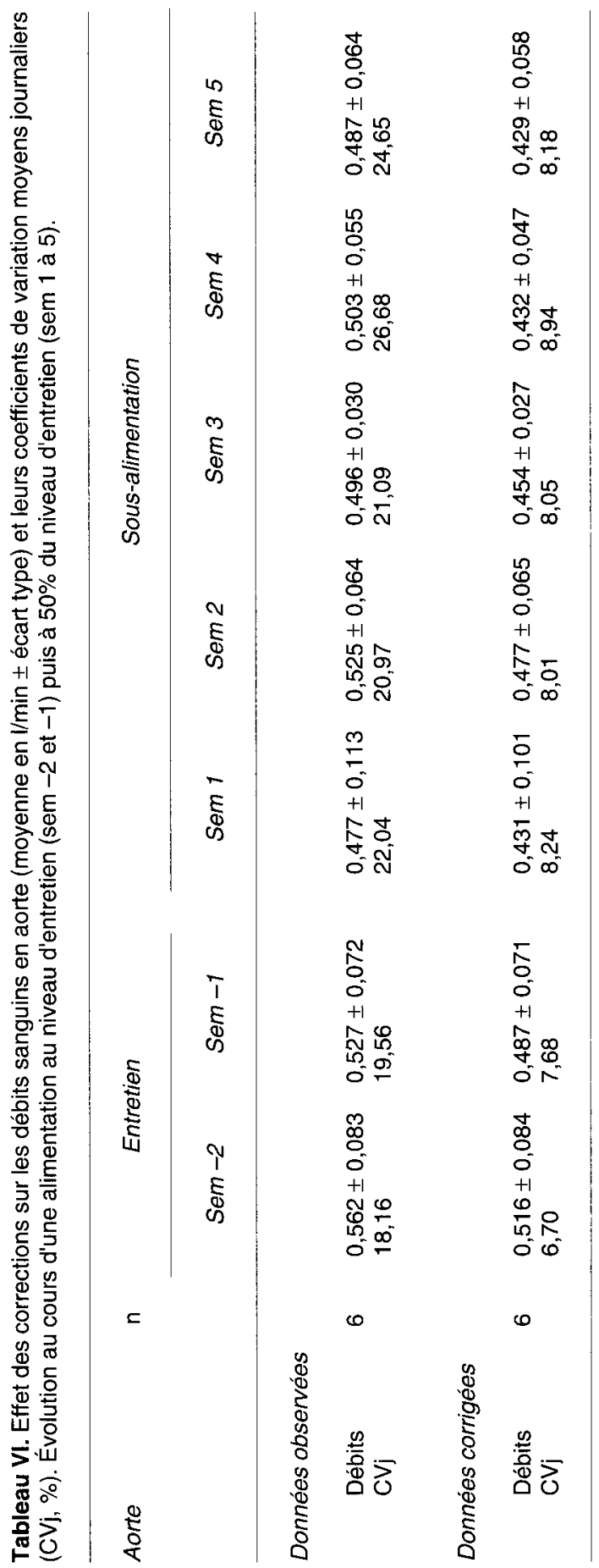


semble des valeurs (tableau IV). De plus $77 \%$ de ces valeurs instantanées éliminées par l'intervalle de confiance correspondaient aux conséquences d'interventions sur les animaux (mouvements de l'expérimentateur avant et après prélèvements pour entrer ou sortir de la cage).

Les débits moyens corrigés sont présentés tableau VI. Les corrections apportées ont baissé les débits aortiques de l'ordre de $9,8 \%$ en moyenne. Cette baisse est significative $(P<0,05)$ pour toutes les semaines de sous-alimentation et la dernière semaine d'entretien (sem-1). La moyenne des $\mathrm{CVj}$ a baissé significativement $(P<0,05)$ de $22 \%(10$ à $44 \%)$ avant correction à $8 \%(4 \%$ à $16 \%)$ après correction. Néanmoins, à l'intérieur de chaque semaine, la variabilité inter-animal n'a pas été réduite de façon significative.

II faut préciser toutefois que le CVj moyen sur les données observées était plus élevé pour l'animal le plus «nerveux» du groupe (O) que pour l'animal le plus "calme» (C). Par ailleurs, la proportion de points supprimés correspondant à des interventions de l'expérimentateur était plus faible pour l'animal "nerveux" $(68 \%)$ que pour l'animal «calme» $(84 \%)$. La «nervosité» de l'animal O a entraîné la suppression de $37 \%$ des valeurs instantanées.

\section{Conséquences des corrections sur l'effet traitement}

La correction des débits Ao a permis de montrer une baisse significative des débits avec la sous-alimentation qui n'apparaissait pas avec les débits observés (tableau $V$ ). Un test $F$ a montré que la variance résiduelle n'a pas été réduite de façon significative.

\section{DISCUSSION}

L'utilisation d'animaux multi-cathétérisés pendant une période aussi longue ( 9 sem chacun au minimum) a montré qu'il était difficile de maintenir tous les cathéters entièrement fonctionnels jusqu'à la fin de l'expérience. Dans nos conditions expérimentales, un maximum de 2 animaux pouvait être conduit simultanément, si bien que 18 mois ont été nécessaires pour mener à bien l'ensemble de l'expérience (Ortigues et Durand, 1994). La lourdeur de ce type d'expérimentation in vivo, où il est difficile de travailler sur un nombre important d'animaux, montre bien l'intérêt d'exploiter au mieux les résultats.

\section{Débits splanchniques}

Au niveau splanchnique, les débits sanguins ont été obtenus grâce à une méthode de dilution. Exprimés par $\mathrm{kg}$ de poids les débits sanguins en VP et en VSH (48 et $54 \mathrm{ml} / \mathrm{min} / \mathrm{kg} \mathrm{PV}$ ) étaient similaires à ceux obtenus par Webster et al (1975) sur moutons. En revanche, ils étaient légèrement supérieurs à ceux notés par Bergman et Wolff (1971), Pethick et al (1981) et Heitmann et al (1986) chez des moutons ou des brebis matures, et par Burrin et al (1989) et Gross et al (1990) chez des agneaux en croissance (34-46 et $38-51 \mathrm{ml} / \mathrm{min} / \mathrm{kg} \mathrm{PV}$, respectivement), utilisant une technique de $\mathrm{PAH}$. La contribution de l'artère hépatique au débit splanchnique total était en moyenne de $12,5 \%$. Chez les ovins, cette contribution varie de 10,4\% (Bergman et Wolff, 1971) à 37\% (Burrin et al, 1989) avec la technique de $\mathrm{PAH}$, mais est de l'ordre de 2 à $5 \%$ lorsque la mesure se fait à l'aide de microsphères (Barnes et al, 1986). Exprimés par kg de poids de tissus (mesurés à l'abattage en fin de période de sous-alimentation (Ortigues et Durand, 1994)), les débits splanchniques s'élevaient à 0,51 , 1,09 et $4,53 \mathrm{ml} / \mathrm{min} / \mathrm{g}$ de tissu frais pour le tube digestif total ou dégraissé et pour le foie, respectivement. Ces résultats sont similaires à ceux obtenus par Burrin et al 
(1989) : 0,72-0,83 $\mathrm{ml} / \mathrm{min} / \mathrm{g}$ tissu frais pour l'ensemble des estomacs et des intestins et $3,48-4,27 \mathrm{ml} / \mathrm{min} / \mathrm{g}$ pour le foie.

La mesure des débits sanguins par dilution, en utilisant le PAH, montre une assez grande variabilité des valeurs de débits instantanés à l'intérieur d'une journée de prélèvements. Nos résultats sont en accord avec ceux observés dans la littérature. Ainsi Ortigues et al (1994) rapportent des CV de $15 \%$ en moyenne sur brebis, Huntington (1982) de 16 à $32 \%$ chez des vaches. Cette variabilité est en partie liée aux incertitudes de dosage, aux déplacements momentanés des cathéters dans les vaisseaux et à un mauvais mélange de $\mathrm{PAH}$ dans le sang (Ortigues et al, 1994).

La perfusion de PAH dans 2 sites a fortement baissé la variabilité associée aux débits moyens journaliers par rapport à l'infusion en VM seule, les CVj n'étaient plus que de $11 \%$ en VP et $12 \%$ en VSH. Les CVj de $11 \%$ et $12 \%$ sont similaires à ceux mesurés par Ortigues et al (1994) chez des brebis dans d'autres conditions nutritionnelles et confirment ainsi le choix de la valeur de référence $(11 \%)$. Ceci démontre bien que les incertitudes méthodologiques ont pu être réduites et que la fiabilité des valeurs de débits VP et VSH a pu être améliorée par l'infusion en 2 sites.

La méthode de correction que nous avons testée a aussi permis de réduire significativement les $C V$ j. Dans le cas de l'infusion en un seul site (en VM), soit par choix expérimental, soit pour une cause de non fonctionnement des cathéters, les incertitudes liées aux aspects méthodologiques peuvent être réduites de manière importante par le biais de corrections. Ainsi les CVj en VP sont passés de $18 \%$ à $14 \%$ et les $\mathrm{CVj}$ en VSH de 17 à $13 \%$ après élimination de 9 et $7 \%$ des valeurs instantanées respectivement. Dans la pratique, des corrections sont souvent apportées mais de manière non rigoureuse et non reproductible. Casse (1990) obtient des CVj de 10\% chez la vache laitière après élimination d'un plus grand nombre de valeurs mesurées $(25 \%)$. Cependant cet auteur ne décrit pas précisément la méthode utilisée pour éliminer les valeurs aberrantes. La méthode présentée ici a l'avantage d'être à la fois objective et reproductible.

Par ailleurs, chez les animaux perfusés en 2 sites, le nombre de valeurs instantanées éliminées a été 2 fois moins important, et les corrections ont porté sur 2 fois moins de journées de mesures que pour les animaux infusés en un seul site. La combinaison d'une infusion de PAH en 2 sites et des corrections permet d'obtenir des $\mathrm{CVj}$ aussi faibles que ceux rapportés par Casse (1990) tout en conservant un plus grand nombre de valeurs (96-97\% au lieu de $75 \%$ seulement).

Il est surprenant de noter que l'infusion de PAH en 2 sites a aussi diminué la variabilité des débits sus-hépatiques pour lesquels le mélange de $\mathrm{PAH}$ dans le sang devrait être amélioré après passage par les capillaires hépatiques. Ce résultat soulève la question de la distribution du sang porte dans le foie. Selon Heath et Perkins (1985), la distribution du sang mésentérique, gastrosplénique et gastroduodénal serait homogène dans les différents lobes du foie d'ovins adultes. En revanche, une hétérogénéité de distribution a été notée par Heath (1968) et Hancock et Milligan (1985), qui pourrait expliquer nos résultats. Cet aspect reste à élucider car il détermine la validité des bilans hépatiques de nutriments obtenus par des méthodes artério-veineuses.

En ce qui concerne les débits de l'AH, ils sont calculés par différence entre les débits de la VSH et ceux de la VP; les erreurs se trouvent donc cumulées, conduisant parfois à des valeurs physiologiquement aberrantes. II est important de noter ici que les corrections apportées ont permis de corriger les valeurs de débits et de contribution de l'AH qui étaient négatives et non physiologiques (Casse, 1990). 
Dans le cadre de l'expérience étudiée, les corrections n'ont modifié ni les valeurs moyennes des débits splanchniques, ni les conclusions de l'analyse de variance concernant les effets de la sous-alimentation et ceux du temps. Néanmoins, la variance résiduelle a été améliorée. Ceci peut s'avérer important dans d'autres expériences.

L'étude présentée ici s'est intéressée aux débits splanchniques moyens dans le but de calculer des bilans journaliers de nutriments (Ortigues et Durand, 1994). Dans le cadre d'études de débits sanguins en cinétique (ex: cinétiques post-prandiales), chaque point est important et ne peut être éliminé d'après la méthode de correction proposée dans cet article. Aussi, la perfusion de PAH en 2 sites est essentielle.

\section{Débits aortiques}

Les débits sanguins aortiques obtenus sont difficiles à comparer à ceux de la littérature car les zones anatomiques mesurées varient en fonction de la préparation chirurgicale utilisée dans chaque expérience. Cependant, exprimés par unité de poids de tissu (mesuré à l'abattage [Ortigues et Durand, 1994]), les débits sanguins des brebis "debout calme" s'élevaient en moyenne à 57,7 ou $79,3 \mathrm{ml} / \mathrm{min} / \mathrm{kg}$ patte arrière ou par kg de patte arrière désossée à la fin de la période de sous-alimentation. Ces valeurs sont similaires à celles mesurées chez des agneaux en croissance $(26-91 \mathrm{ml} / \mathrm{min} / \mathrm{kg}$ patte arrière [Harris et al, 1992]) mais plus faibles que chez des brebis taries debout (70-132 $\mathrm{ml} / \mathrm{min} / \mathrm{kg}$ patte-arrière [Oddy et al, 1984]). Ces derniers auteurs n'ont pas tenu compte de l'activité physique des animaux.

Au niveau du train-arrière, nos résultats démontrent l'importance de prendre en compte l'activité physique spontanée des animaux dans les mesures de débits sanguins afin de comparer les animaux entre eux dans un même état d'activité physique.
Ces corrections ne s'appliquent néanmoins qu'à des animaux initialement sélectionnés pour leur caractère calme, déjà habitués à l'environnement, aux prélèvements et qui ne présentent qu'un agitation mineure. Elles ne concernent aussi que les périodes d'agitation visible où les débits sanguins peuvent être multipliés par 2 ou 3 lors d'une agitation particulière, même faible. Ces corrections ne prennent pas en compte l'état de stress ou de tension nerveuse des différents animaux. Chez des animaux nerveux ou stressés dont l'agitation serait de longue durée ou très fréquente, les bilans de métabolites obtenus avec des débits corrigés seraient ininterprétables. En effet, les débits élevés et les différences artériovéneuses en métabolites seraient alors représentatifs d'un métabolisme musculaire en état d'activité physique ou de stress et non de l'influence de facteurs alimentaires sur ce métabolisme.

Par ailleurs, nos résultats montrent que les interventions sur les animaux au moment des prélèvements doivent être notées, car elles ont une influence importante sur les débits ( $77 \%$ des valeurs supprimées par les corrections étaient liées à l'entrée de l'expérimentateur dans la cage avant les prélèvements ou à sa sortie par la suite). Pendant les prélèvements les animaux restaient calmes, ce qui justifiait d'utiliser les débits corrigés pour le calcul des bilans de métabolites. Un système de prélèvement à distance serait souhaitable ; néanmoins, il accroîtrait les durées de prélèvements et les risques de mauvais fonctionnement des cathéters. Enfin, la saisie intégrée des résultats sur micro-ordinateur semble nettement préférable à un enregistrement sur papier suivi d'une lecture manuelle des débits toutes les minutes.

Contrairement à ce qui a été rapporté au niveau splanchnique, les corrections ont permis de montrer un effet significatif de la sous-alimentation sur les débits au niveau du train-arrière, alors que cette réponse 
n'avait pas pu être mise en évidence sur les débits observés.

La correction appliquée a permis d'évaluer les débits sanguins d'animaux «debouts et calmes" pendant toute la durée de l'expérimentation. Ces corrections s'avèrent essentielles afin d'éviter que l'activité physique des animaux ne biaise les comparaisons entre traitements. La méthode de dépouillement présentée pour les débits du train-arrière a les mêmes rigueur et reproductibilité que celles proposées pour les débits splanchniques.

\section{RÉFÉRENCES}

Barnes RJ, Comline RS, Dobson A (1986) The control of splanchnic blood flow. In : Control of Digestion and Metabolism in Ruminants (Milligan LP, Grounm WL, Dobson A, eds), Prentice Hall, Englewood Cliffs, NJ, $41-59$

Bergman EN, Wolff JE (1971) Metabolism of volatile fatty acids by liver and portal-drained viscera in sheep. Am J Physiol 221, 586-592

Burrin DG, Ferrell CL, Eisemann JH, Britton RA, Nienaber JA (1989) Effect of level of nutrition of splanchnic blood flow and oxygen consumption in sheep. Br J Nutr 62, 23-34

Casse $E$ (1990) Métabolisme des nutriments énergétiques dans le foie des vaches laitières hautes productrices. Thèse, INA Paris-Grignon

Gross KL, Harmon DL, Avery TB (1990) Portal-drained visceral flux of nutrients in lambs fed alfalfa or maintained by total intragastric infusion. J Anim Sci 68, 214-221

Hancock MJ, Milligan LP (1985) Distribution of portal blood to the liver of sheep. J Anim Sci61 (suppl 1), 454-455

Harris PM, Garlick PJ, Lobley GE (1989) Interactions between energy and protein metabolism in the whole body and hind limb of sheep in response to intake. In: Energy Metabolism of Farm Animals (Van der Honing $Y$, Close WH, eds), Pudoc, Wageningen, 167-170
Harris PM, Skene PA, Buchan V et al (1992) Effect of food intake on hind-limb and whole-body protein metabolism in young growing sheep: chronic studies based on arterio-venous techniques. $\mathrm{Br} J \mathrm{Nutr}$ $68,389-407$

Heath T (1968) Origin and distribution of portal blood in the sheep. Am J Anat 22, 95-106

Heath T, Perkins NR (1985) Effect of development of the ovine forestomachs on the anatomy of portal vessels and on the intrahepatic distribution of portal blood. Res Vet Sci 39, 216-221

Heitmann RN, Sensenig SC, Reynolds CK, Fernandez JM, Dawes DJ (1986) Changes in energy metabolite and regulatory hormone concentrations and net fluxes across splanchnic and peripheral tissues in fed and progressively fasted ewes. J Nutr 116, 25162524

Huntington GB (1982) Portal blood flow and net absorption of ammonia-nitrogen, and glucose in nonlactating Holstein cows. J Dairy Sci 65, 1155-1162

Katz ML, Bergman EN (1969) Simultaneous measurements of hepatic and portal venous blood flow in the sheep and dog. Am J Physiol 216, 946-952

Oddy VH, Gooden JM, Annison EF (1984) Partitioning of nutrients in Merinos ewes. I. Contribution of skeletal muscle, the pregnant uterus and the lactating mammary gland to total energy expenditure. Austr J Biol Sci 37, 375-388

Ortigues I (1991) Adaptation du métabolisme énergétique des ruminants à la sous-alimentation. Quantification au niveau de l'animal entier et des tissus corporels. Reprod Nutr Dévelop 31, 593-616

Ortigues I, Durand D (1994) Adaptation of energy metabolism to undernutrition in ewes. Contribution of portal drained viscera, liver and hindquarters. Br J Nutr (sous presse)

Ortigues I, Durand D, Lefaivre J (1994) Use of paraamino-hippuric acid to measure blood flows through portal drained viscera, liver and hindquarters in sheep. J Agr Sci Camb 122, 299-308

Pethick DW, Lindsay DB, Barker PJ, Northrop AJ (1981) Acetate supply and utilization by the tissues of sheep in vivo. Br J Nutr 46, 97-110

Sokal RR, Rohlf FJ (1989) Biometry. WH Freeman and Co, San Francisco

Webster AJF, Osuji PO, White F, Ingram JF (1975) The influence of food intake on portal blood flow and heat production in the digestive tract of sheep. Br J Nutr $33,425-438$ 